\title{
晶场繴裂序参量导致稀土-硼十二化合物中磁性和 比热反常的理论研究
}

\author{
胡靖三 ${ }^{1}$, 顾建飞 1 , 章维益 ${ }^{1,2}$ \\ (南京大学 1. 物理学院; 2. 固体微结构国家重点实验室, 南京 210093)
}

摘 要: 在低温条件 $\left(T_{\mathrm{C}} \approx 3 \sim 22 \mathrm{~K}\right)$ 下, 除了 $4 \mathrm{f}$ 电子满壳层的 $\mathrm{LuB}_{12}$ 之外, 其他稀土-硼十二化合物(简称 $\mathrm{RB}_{12}, \mathrm{R}$ 为稀 土元素 Tb, Dy, Ho, Er, Tm)存在磁性和比热的反常现象, 该反常现象的机制至今仍存争议。尽管磁化率曲线上的隆 起与 $\mathrm{R}^{3+}$ 离子的反铁磁序类似, 但是中子衍射谱和莫斯堡尔谱的实验均没有明确给出稀土离子的磁结构。这些化合 物磁化率、熵的反常行为和低温中子谱的结果都与 $\mathrm{RB}_{12}$ 具有幅度调制的磁结构一致。本课题组采用模型和数值计 算相结合的方法来探究该反常行为的物理机制。研究表明：由于稀土离子 $\mathrm{meV}$ 量级的晶场䢃裂易受热涨落扰动，晶 场䢃裂能不再是一个量子力学参量, 而是一个热力学序参量。物理图像中轨道角动量逐渐淬灭很好地解释了磁性和 比热的反常现象。结合该类晶体低温下在(111)晶向的二聚化行为和非弹性散射谱的结果, 对中子散射谱中的双峰结构 也给予了一个可能的解释。

关 键 词: 稀土化合物; 固体磁性; 晶场䢃裂; 序参量

中图分类号: TM28 文献标志码: A

\section{Mechanism of the Magnetic and Specific-heat Anomalies in Rare-earth Dodecaborides $R_{12}(R=T b-T m)$ : an Effect of Crystal-field-splitting Order Parameter}

\author{
HU Jingsan ${ }^{1}$, GU Jianfei ${ }^{1}$, ZHANG Weiyi ${ }^{1,2}$ \\ (1. School of Physics, Nanjing University, Nanjing 210093, China; 2. National Laboratory of Solid State Microstructures and \\ Department of Physics, Nanjing University, Nanjing 210093, China)
}

\begin{abstract}
Rare-earth dodecaborides $\mathrm{RB}_{12}(\mathrm{R}=\mathrm{Tb}$, Dy, Ho, Er, $\mathrm{Tm})$ are all characterized by a controversial and unresolved magnetic and specific-heat anomaly at low temperature $\left(T_{\mathrm{C}} \approx 3-22 \mathrm{~K}\right)$ except $\mathrm{LuB}_{12}$ with closed f-shell. Although the bump feature in magnetic susceptibilities resembles that of antiferromagnetically ordered $\mathrm{R}^{3+}$ ions, no definite magnetic structure was identified by either neutron scattering or Mössbauer spectra. The anomalies in susceptibilities, entropy, and low temperature neutron diffraction pattern all pointed to the amplitude-modulated nature of complex magnetic structure. In view of the rather small crystal-field-splitting energies of the order of meV, we propose to treat crystal-field-splitting (CFS) energy as an order parameter rather than a quantum mechanical quantity. In this way, we found that not only the magnetic and specific-heat anomalies can be explained properly by a gradual
\end{abstract}

收稿日期：2020-11-03; 收到修改稿日期：2021-01-18; 网络出版日期：2021-03-01

基金项目: 国家重点研发计划(2017YFA0303202)

National Key Research and Development Program of China (2017YFA0303202)

作者简介：胡靖三(1994-), 男，博士研究生. E-mail: b111120046@smail.nju.edu.cn

HU Jingsan (1994-), male, PhD candidate. E-mail: b111120046@smail.nju.edu.cn

通信作者: 章维益，教授. E-mail: wyzhang@nju.edu.cn

ZHANG Weiyi, professor. E-mail: wyzhang@nju.edu.cn 
quenching of $\mathrm{R}$ orbital moments, but also the low temperature satellite peaks of neutron scattering spectra can be understood in terms of the spontaneously (111) dimerised structure and inelastically absorbed rattling phonon modes.

Key words: rare-earth compound; magnetism in solid; crystal-electrical-splitting; order parameter

近年来, 稀土元素 $(R)$ 的嗍化物 $R_{12}$ 由于新颖 的物理化学性质和广阔的应用前景, 吸引了研究人 员的广泛关注 ${ }^{[1-2]}$ 。在芯片产业和自旋电子学蓬勃发 展的今天, 稀土化合物作为核心支柱材料之一, 研 究它们的磁性具有重要的意义。 $R_{12}$ 众多新奇的磁 学现象主要源自不同的稀土元素, 例如: $\mathrm{LuB}_{12}$ 是临 界温度 $T_{\mathrm{C}}=0.4 \mathrm{~K}$ 的超导体 ${ }^{[3]}, \mathrm{YbB}_{12}$ 是近藤温度 $T_{K} \approx 70 \mathrm{~K}$ 的近藤绝缘体 ${ }^{[4]}$ 。

在 $\mathrm{NaCl}$ 型立方结构的 $\mathrm{RB}_{12}$ 晶体中, 稀土离子 $\mathrm{R}$ 和 $\mathrm{B}_{12}$ 分别占据不同的格点 ${ }^{[5-6]}$ 。Menushenkov 等 ${ }^{[7]}$ 发现，在低温相 $\mathrm{RB}_{12}(\mathrm{R}=\mathrm{Ho}, \mathrm{Er}, \mathrm{Tm}, \mathrm{Yb}, \mathrm{Lu})$ 系列材 料中, 稀土原子相对于高温相高对称位置总会发生 $0.02 \sim 0.03 \mathrm{~nm}$ 的偏离。这种晶格畸变使得 $\mathrm{RB}_{12}$ 的理 想面心立方结构只在高温下存在 ${ }^{[8]}$ 。在拉曼散射实 验中, Werheit 等 ${ }^{[9]}$ 发现稀土离子在 $\mathrm{B}_{24}$ 组成的截角 八面体笼子内拥有多个等价双势阱位置。这种独特 的双势阱结构使得稀土离子具备不同尺度的两种振 动模式 ${ }^{[7]}$ 。Moiseenko 等 ${ }^{[10]}$ 通过 90 1200 K 温区 $\mathrm{RB}_{12}$ 高温磁化率的测量, 证实稀土离子满足完美的顺磁 盐行为。通过外延得到负的居里温度似乎暗示着稀 土离子的某种反铁磁序。当温度下降到 $3 \sim 20 \mathrm{~K}$ 时, Gabáni 等 ${ }^{[11]}$ 发现在 $\mathrm{RB}_{12}(\mathrm{R}=\mathrm{Dy}, \mathrm{Ho}, \mathrm{Er}, \mathrm{Tm})$ 的磁化 率曲线上出现了一个反常峰。在相同的温区, Czopnik 等 ${ }^{[12]}$ 观测到 $R_{12}(R=T b, D y, H o, E r, T m)$ 的比热 曲线同样出现狭窄而尖锐的峰。科研人员认为这个 相变是稀土离子的反铁磁一顺磁相变造成的, 但是 稀土离子磁矩的取向序不能完全解释比热曲线近乎 垂直的陡峭下降。比热 ${ }^{[12]}$ 、莫斯堡尔谱 ${ }^{[13]}$ 和磁电阻 实验 ${ }^{[14-15]}$ 都证明, 稀土离子的总角动量在磁性反常 前后发生了变化。Moiseenko 等 ${ }^{[10]}$ 尝试采用自由电 子的 RKKY 相互作用模型来解释反铁磁的产生, 但 是 RKKY 模型依赖具体的晶体结构和电子能带结 构, 费米面附近复杂的多能带结构并非完全支持反 铁磁间接耦合图像 ${ }^{[16]}$ 。另外与理想的反铁磁结构不 同, 这些材料在衍射面指数 $(1 / 2,1 / 2,1 / 2)$ 附近都出现 成对的中子散射双峰结构 ${ }^{[16-19]}$ 。综上所述, 迄今为 止的中子衍射谱和莫斯堡尔谱都没能给出稀土化合 物中明确的磁结构, 因此 $\mathrm{RB}_{12}$ 中的低温磁化率和比 热反常现象仍然是一个县而未决的问题。

受到稀土离子总角动量大小在反常峰前后改变 的启发, 可以认为这一系列材料的低温反常行为与
晶场和热涨落的竞争有关。众所周知, 过渡金属 $3 \mathrm{~d}$ 轨道的晶场䢃裂能在 $\mathrm{eV}$ 量级, 常温下几乎不受热 涨落影响, 可以将它作为一个材料系数对待(见图 1(a) 所示)。稀土离子的 $4 \mathrm{f}$ 晶场䢃裂能比过渡金属的 $3 \mathrm{~d}$ 晶场䢃裂能小两个量级, 因此热涨落导致的晶格振 动对晶场的影响不容忽视。为了解释稀土化合物的 低温磁化率和比热的反常现象, 本研究提出以下物 理机制(如图 1(b)): 在远离磁化率比热反常峰的低 温区间, 稀土离子的晶场䢃裂能远小于自旋-轨道 耦合能, 远大于热振动带来的能量涨落, 轨道磁矩 部分淬灭。随着温度升高, 热涨落导致的晶格振动 幅度增大, 晶场的各向异性减弱, 从而晶场䢃裂能 变小。最终在临界温度, 热振动导致晶场趋于各向 同性, 热涨落使晶场能级完全模糊, 轨道磁矩得到 完全释放。上述讨论说明, 不能把稀土离子的微小 晶场䢃裂看作一个量子力学常量, 而将其视作随温 度变化的一个热力学序参量。序参量减小的过程也 是轨道磁矩逐渐释放的过程。当温度升至临界温度 及以上，稀土离子的晶场䢃裂能序参量彻底消失， 稀土离子回归到由洪特三定则描述的顺磁离子。这 就是磁化率比热反常中磁矩幅度变化的物理机制。 关于低温中子衍射谱中的双峰结构, 本课题组将尝 试通过 $\mathrm{B}_{24}$ 截角八面体笼子内的双势阱导致的晶格 二聚化和两种爱因斯坦晶格振动模式加以解释。

\section{1 计算方法}

\section{1 晶场䢃裂能}

稀土离子 $4 \mathrm{f}$ 电子的能级远低于 $\mathrm{RB}_{12}$ 金属的费 米能，而且与周围硼原子的耦合也很弱，可以分离 出稀土离子的有效哈密顿量。在忽略了多带传导电 子引起的稀土离子间微小的 RKKY 耦合后, 晶场中 稀土离子的顺磁哈密顿量由自旋轨道耦合能 $H_{\mathrm{SO}}$ 、
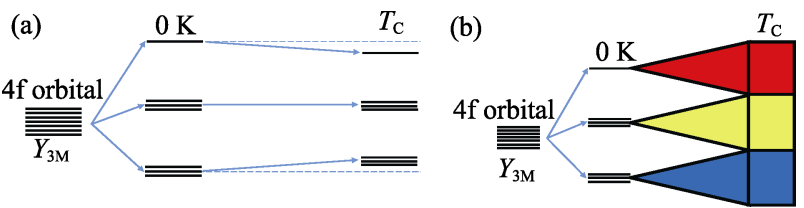

图 1 稀土离子 $4 \mathrm{f}$ 轨道能级示意图

Fig. 1 Schematic diagram of crystal-levels

(a) Crystal-field-splitting energy reduction due to thermal expansion; (b) Crystal-levels broadening due to thermal lattice fluctuation 
晶场䢃裂能 $H_{\mathrm{CF}}$ 和塞曼繴裂能 $H_{\mathrm{ZS}}$ 三种相互作用竞 争而成 ${ }^{[20-21]}$, 可以表示为:

$$
H_{0}=H_{\mathrm{SO}}+H_{\mathrm{CF}}+H_{\mathrm{ZS}}
$$

其中 $H_{\mathrm{SO}}^{i}=\xi \overrightarrow{l_{i}} \cdot \overrightarrow{s_{i}}=0.5\left[j_{i}\left(j_{i}+1\right)-l(l+1)-s(s+1)\right]$ 描 述了 $4 \mathrm{f}$ 壳层中第 $i$ 个电子的自旋和轨道角动量平行 或反平行排列的能量, $\xi \approx 0.2 \sim 0.4 \mathrm{eV}^{[21]}$ 为自旋轨 道耦合常数。这一项在哈密顿量中占主导地位, 它 的矩阵元在单电子的总角动量空间 $\mid j m_{j}$ 中是对角 的。对稀土的 $4 \mathrm{f}$ 壳层来说, $l=3, s=1 / 2$, 所以对角 矩阵元为 $1.5 \xi$ (八重态 $j=7 / 2$ ) 或 $-2 \xi$ (六重态 $j=5 / 2)$ 。考虑到晶场䢃裂能, 同样可以在轨道 $Y_{\mathrm{lm}}$ 和自旋 $\sigma= \pm 1$ 的直积空间 $\mid Y_{\operatorname{lm}} \sigma$ 中表达单电子的自 旋轨道耦合哈密顿量, 它的矩阵元为:

$$
\begin{aligned}
& Y_{\mathrm{lm}^{\prime}} \sigma^{\prime}\left|H_{\mathrm{SO}}\right| Y_{\mathrm{lm}} \sigma=0.5 \xi[\sqrt{(l-\sigma m)(l+\sigma m+1)} \\
& \left.\delta_{m^{\prime} m+\sigma} \delta_{\sigma^{\prime} \bar{\sigma}}+\sigma m \delta_{m^{\prime} m} \delta_{\sigma^{\prime} \sigma}\right]
\end{aligned}
$$

其次, 假定磁场 $\vec{B}$ 取 $\vec{n}=\left(n_{x}, n_{y}, n_{z}\right)$ 方向, 塞曼䢃裂 项 $H_{\mathrm{ZS}}=-\mu_{\mathrm{B}} \vec{B}[\vec{l}+2 \vec{s}]$ 的矩阵元可写为:

$$
\begin{aligned}
& \left\langle Y_{\mathrm{lm}^{\prime}} \sigma^{\prime}\left|H_{\mathrm{ZS}}\right| Y_{\mathrm{lm}} \sigma\right\rangle=-\mu_{\mathrm{B}} B . \\
& {\left[0.5\left(n_{x}-i n_{y}\right) \sqrt{(l-m)(l+m+1)} \delta_{m^{\prime} m+1} \delta_{\sigma^{\prime} \sigma}+\right.} \\
& 0.5\left(n_{x}+i n_{y}\right) \sqrt{(l+m)(l-m+1)} \delta_{m^{\prime} m-1} \delta_{\sigma^{\prime} \sigma}+ \\
& \left.\left(n_{x}+i \sigma n_{y}\right) \delta_{m^{\prime} m} \delta_{\sigma^{\prime} \bar{\sigma}}+n_{z}(m+\sigma) \delta_{m^{\prime} m} \delta_{\sigma^{\prime} \sigma}\right]
\end{aligned}
$$

最后, $\mathrm{RB}_{12}$ 中 $\mathrm{R}^{3+}$ 离子处在立方晶场中, 最近邻为 6 个由 $\mathrm{B}_{12}$ 形成的截角立方体。它的晶场波函数 $f_{i}(i=1 \sim 7)$ 分别为 $f_{x y z}=\left(Y_{32}-Y_{3 \overline{2}}\right) / \sqrt{2}, f_{x\left(y^{2}-z^{2}\right)}=$ $\left(\sqrt{3} Y_{33}+\sqrt{5} Y_{31}-\sqrt{5} Y_{3 \overline{1}}-\sqrt{3} Y_{3 \overline{3}}\right) / 2, \quad f_{y\left(z^{2}-x^{2}\right)}=\left(\sqrt{3} Y_{33}-\right.$ $\left.\sqrt{5} Y_{31}-\sqrt{5} Y_{3 \overline{1}}-\sqrt{3} Y_{3 \overline{3}}\right) / 2, \quad f_{z\left(x^{2}-y\right)}=\left(Y_{32}+Y_{3 \overline{2}}\right) / \sqrt{2}$, $f_{x\left(5 x^{2}-3 r^{2}\right)}=\left(\sqrt{5} Y_{33}-\sqrt{3} Y_{31}+\sqrt{3} Y_{3 \overline{1}}-\sqrt{5} Y_{3 \overline{3}}\right) / 2, \quad f_{y\left(5 y^{2}-3 r^{2}\right)}=$ $\left(\sqrt{5} Y_{33}+\sqrt{3} Y_{31}+\sqrt{3} Y_{3 \overline{1}}+\sqrt{5} Y_{3 \overline{3}}\right) / 2$ 和 $f_{z\left(5 z^{2}-3 r^{2}\right)}=Y_{30}$ 。 晶场䢃裂哈密顿量可用晶场波函数写为:

$$
H_{\mathrm{CF}}=\sum_{i=1, \sigma}^{7} J_{i} f_{i \sigma}^{\dagger} f_{i \sigma}
$$

其中 $J_{i}$ 为晶场䢃裂轨道能量修正, $f_{i \sigma}$ 为单电子晶 场态 $i$ 和自旋 $\sigma$ 的湮灭算符。在立方晶场下, $f$ 电子 擘裂为 1 个单重态 $\left(J_{1}=0\right)$ 和 2 个三重态 $\left(J_{2}=J_{3}=\right.$ $\left.J_{4}=\Delta_{1}, J_{5}=J_{6}=J_{7}=\Delta_{2}\right)$ 。晶场能级的䢃裂由两 个参数 $\Delta_{1}$ 和 $\Delta_{2}$ 描述。对于一个 $+Z$ 价的 $\mathrm{R}^{Z+}$ 离子来 说, 其 $4 \mathrm{f}$ 电子的晶场势可以用点电荷模型的静电势 估算:

$$
\begin{gathered}
V(\vec{r})=\frac{\alpha e^{2}}{4 \pi \epsilon_{0} a}+\frac{280 Z e^{2}}{4 \pi \epsilon_{0} a^{5}}\left[\left(x^{4}+y^{4}+z^{4}\right)-\frac{3}{5} r^{4}\right]- \\
\frac{1344 Z e^{2}}{4 \pi \epsilon_{0} a^{7}}\left[\left(x^{6}+y^{6}+z^{6}\right)+\frac{15}{4}\left(x^{2} y^{4}+y^{2} z^{4}+\right.\right. \\
\left.\left.z^{2} x^{4}+x^{2} z^{4}+y^{2} x^{4}+z^{2} y^{4}\right)-\frac{15}{14} r^{6}\right]
\end{gathered}
$$

公式(5)中的晶场势由三部分组成: 第一项给出与电 子位置无关的点能量(马德隆能量)。当 $Z=3$ 时, 马 德隆常数 $\alpha=10.4856$ 。与电子位置有关的短程 4 次

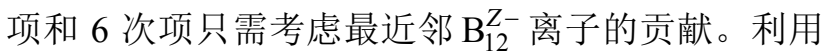
公式(5)和上面给出的晶场波函数, 可以得到点电荷 模型下的两个晶场䢃裂参数:

$$
\begin{aligned}
& \Delta_{1}^{0}=\frac{2 Z \mathrm{Z}^{2}}{4 \pi \epsilon_{0} a}\left[\frac{560}{33} \frac{\left\langle r^{4}\right\rangle}{a^{4}}-\frac{6720}{143} \frac{\left\langle r^{6}\right\rangle}{a^{6}}\right] \\
& \Delta_{2}^{0}=\frac{2 Z \mathrm{e}^{2}}{4 \pi \epsilon_{0} a}\left[\frac{336}{11} \frac{\left\langle r^{4}\right\rangle}{a^{4}}-\frac{2240}{143} \frac{\left\langle r^{6}\right\rangle}{a^{6}}\right]
\end{aligned}
$$

利用表 1 中的晶体结构参数 ${ }^{[22]}$ 以及 $4 \mathrm{f}$ 轨道半径的 4 次和 6 次矩 $\left\langle r^{4}\right\rangle,\left\langle r^{6}\right\rangle^{[23]}$, 将 $Z=3$ 时估算的晶场䢃 裂参数也列入表 1 中。可以看到, 稀土离子 $4 \mathrm{f}$ 轨道 的晶场䢃裂能确实比过渡金属 $3 \mathrm{~d}$ 轨道小两个量级。

\section{2 晶格振动}

接着考虑晶格热振动带来的局域晶场势的扰 动。由于稀土离子的 $4 \mathrm{f}$ 轨道占据均大于半满, 采用 空穴模型更为方便。假定晶格热振动导致的晶场势 涨落均匀分布在 $N$ 个空穴上, 局域的马德隆能量涨 落引起的能级展宽为:

$$
\Delta V=\frac{Z}{N} \frac{\alpha^{\prime} \mathrm{e}^{2}}{4 \pi \epsilon_{0}} \frac{2}{\sqrt{3}} \frac{Q}{a^{2}}
$$

其中 $Q$ 代表晶格的膨胀-收缩热振动模式的振幅。考 虑到晶格振动导致的无序， $\alpha^{\prime}$ 和公式(5)中的 $\alpha$ 并 不相同， $\alpha^{\prime}$ 应该处在完美晶体的马德隆常数 $\alpha$ 和仅 考虑最近邻离子静电能贡献时的 $\alpha^{\prime}=12 \mathrm{Z}$ 之间。

令能级展宽与晶场䢃裂能相等 $\left(\Delta V=\Delta_{2}^{0}\right)$ 时的 温度为临界温度 $T_{\mathrm{C}}$, 德拜模型给出的临界温度为:

表 $1 \mathbf{R B}_{12}$ 的晶格常数、 $\left\langle r^{n}\right\rangle$ 和晶场擘裂能

Table 1 Lattice constant, $\left\langle r^{n}\right\rangle$ of rare-earth $R$ and crystal-field-splitting energy of $\mathbf{R B}_{12}$

\begin{tabular}{clllll}
\hline $\mathrm{RB}_{12}$ & $\mathrm{TbB}_{12}$ & $\mathrm{DyB}_{12}$ & $\mathrm{HoB}_{12}$ & $\mathrm{ErB}_{12}$ & $\mathrm{TmB}_{12}$ \\
\hline$a /(0.1 \mathrm{~nm})$ & 7.503 & 7.500 & 7.491 & 7.483 & 7.475 \\
$\left\langle r^{4}\right\rangle /\left(\times 10^{-4}, \mathrm{~nm}^{4}\right)$ & 0.1295 & 0.1180 & 0.1081 & 0.09959 & 0.09206 \\
$\left\langle r^{6}\right\rangle /\left(\times 10^{-6}, \mathrm{~nm}^{6}\right)$ & 0.1505 & 0.1328 & 0.1181 & 0.1058 & 0.09530 \\
$\Delta_{1}^{0} / \mathrm{meV}$ & 7.53 & 6.36 & 6.36 & 5.90 & 5.49
\end{tabular}




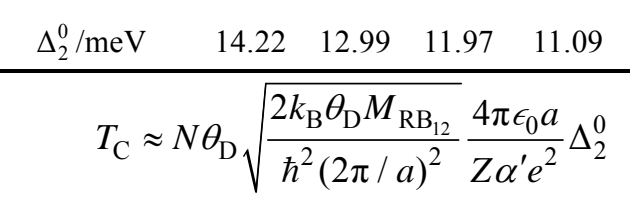

其中 $\theta_{\mathrm{D}} \propto\left(M_{\mathrm{RB}_{12}} a\right)^{-\frac{1}{2}}$ 给出离子模型下不同化合物 的德拜温度与离子质量和晶格常数间的近似关系。

考虑到传导电子对 $4 \mathrm{f}$ 局域电子轨道矩 $\left\langle r^{n}\right\rangle$ 的影 响, 引入下列轨道矩的系统修正:

$$
\left\langle r_{i}^{n}\right\rangle \rightarrow\left[1+\beta \frac{a_{i}-a_{\mathrm{Tm}}}{\sqrt[n]{\left\langle r_{i}^{n}\right\rangle}}+\gamma\right]^{n}\left\langle r_{i}^{n}\right\rangle
$$

其中 $n=4,6$ 。当 $\beta=2.4, \gamma=-0.02$ 时, 得到与实验观 测趋势一致的临界温度。

\section{3 磁化率和比热}

如图 2(a)所示, 所研究的晶场中的稀土离子均 为多空穴体系。以单电子模型的能级图 ${ }^{[24]}$ 为基础, 可以计算多空穴稀土离子的磁化率和比热。对于由 $N$ 个空穴组成的多粒子态 $I$, 离子能量为 $E_{I}^{N}=$ $\sum_{i \in I}^{N}\left\langle N_{I}\left|H_{0}^{i}\right| N_{I}\right\rangle=\sum_{i \in I}^{N} \epsilon_{i}$, 离子磁矩为 $\mu_{I}^{N}=\sum_{i \in I}^{N}\left\langle i\left|\vec{n}\left(\vec{l}_{i}+2 \overrightarrow{s_{i}}\right)\right| i\right\rangle$ 。 由此 $\mathrm{RB}_{12}$ 的顺磁磁化率和比热分别为

$$
\begin{gathered}
\chi_{n}^{N}=\frac{\mu_{\mathrm{B}}}{B} \frac{\sum_{I}^{C_{14}^{N}} \mu_{I}^{N} \exp \left(-E_{I}^{N} / k_{\mathrm{B}} T\right)}{\sum_{I}^{C_{14}^{N}} \exp \left(-E_{I}^{N} / k_{\mathrm{B}} T\right)} \\
\left.C_{\mathrm{V}}^{N}=\frac{1}{k_{\mathrm{B}} T^{2}}\left\{\frac{\sum_{I}^{C_{14}^{N}}\left(E_{I}^{N}\right)^{2} \exp \left(-E_{I}^{N} / k_{\mathrm{B}} T\right)}{\sum_{I}^{C_{14}^{N}} \exp \left(-E_{I}^{N} / k_{\mathrm{B}} T\right)}-\left[\frac{\sum_{I}^{C_{14}^{N}} E_{I}^{N} \exp \left(-E_{I}^{N} / k_{\mathrm{B}} T\right)}{\sum_{I}^{C_{14}^{N}} \exp \left(-E_{I}^{N} / k_{\mathrm{B}} T\right)}\right]^{2}\right]\right\}
\end{gathered}
$$

在下面的讨论中, 把晶场䢃裂能当作序参量 引入进哈密顿中, 这样比热和磁化率的反常将自然 出现。

\section{2 结果和讨论}

经上述讨论, 只需知道 $\mathrm{RB}_{12}$ 晶体的德拜温度就 能估算出不同材料的晶场䢃裂能。迄今为止的实验 只间接测量了 $\mathrm{LuB}_{12}$ 的德拜温度 ${ }^{[3,12]}\left(\theta_{\mathrm{D}} \approx 368 \mathrm{~K}\right)$, 可以通过分子质量和晶格常数估算出其他硼化物 的数据。当 $\alpha^{\prime}=28$ 时, 拟合的晶场䢃裂能和相变温 度与实验数据 ${ }^{[12]}$ 最为接近。这些拟合的最终结果见
表2。
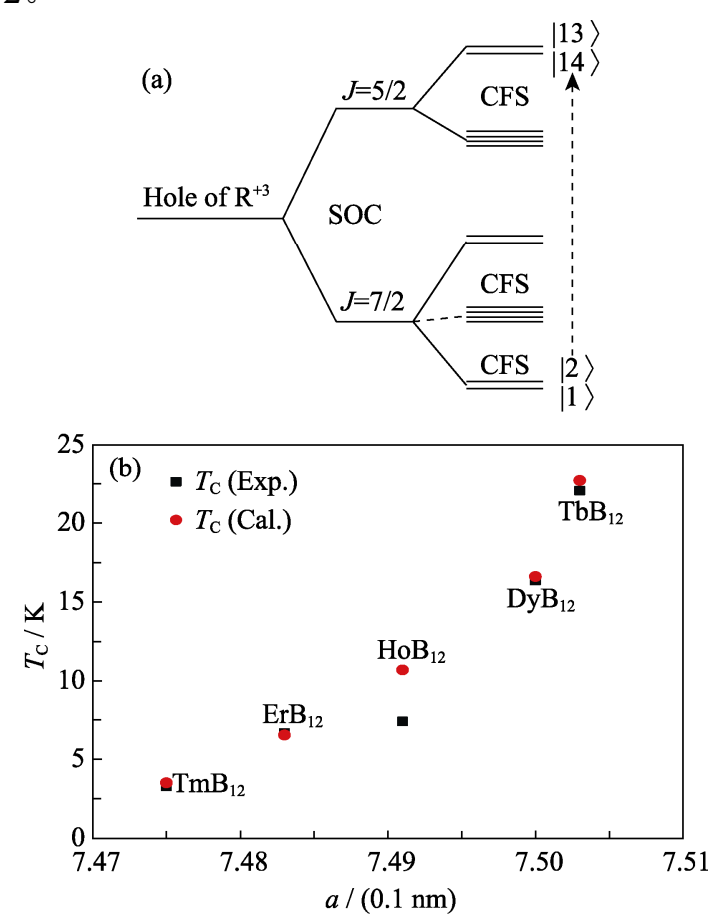

图 2 顺磁稀土离子中单空穴的能级䢃裂示意图(a)和 $\mathrm{RB}_{12}$ 系列的临界温度拟合图(b)

Fig. 2 Level scheme of $4 \mathrm{f}$-orbitals of rare-earth ions in hole picture (a), and the measured and fitted $T_{\mathrm{C}}$ of $\mathrm{RB}_{12}(\mathrm{~b})$

表 $2 \mathbf{R B}_{12}$ 的德拜温度、修正后的晶场䢃裂能和相变温度

Table 2 Debye temperature, corrected crystal-field-splitting parameters, and transition temperature of $\mathbf{R B}_{12}$

\begin{tabular}{cccccc}
\hline $\mathrm{RB}_{12}$ & $\theta_{\mathrm{D}} / \mathrm{K}$ & $\Delta_{1}^{0} / \mathrm{meV}$ & $\Delta_{2}^{0} / \mathrm{meV}$ & $T_{\mathrm{C}}($ Cal. $) / \mathrm{K}$ & $T_{\mathrm{C}}(\operatorname{Exp}.) / \mathrm{K}^{[12]}$ \\
\hline $\mathrm{TbB}_{12}$ & 377 & 10.66 & 20.21 & 22.69 & 22.05 \\
$\mathrm{DyB}_{12}$ & 375 & 9.42 & 17.81 & 16.61 & 16.35 \\
$\mathrm{HoB}_{12}$ & 374 & 7.63 & 14.38 & 10.69 & 7.38 \\
$\mathrm{ErB}_{12}$ & 372 & 6.24 & 11.74 & 6.52 & 6.65 \\
$\mathrm{TmB}_{12}$ & 371 & 5.07 & 9.51 & 3.51 & 3.28 \\
\hline
\end{tabular}

在图 2(b)中根据公式(9)给出了不同稀土-硼十 二化合物的临界温度。除了 $\mathrm{HoB}_{12}$ 外, 其他材料的 误差都在 $3 \%$ 以内。 $\mathrm{Ho}^{3+}$ 离子的偏差是由于其处在半 满的四重态中, Jahn-Teller 效应增强, 进一步提高了 晶场䢃裂能。

\section{1 磁化率和比热}

关于晶场擘裂能序参量的温度依赖关系, 采用 一个类 BCS 弱耦合超导理论的序参量 $\Delta_{i}(T)=$ $\Delta_{i}^{0} \tanh \left\{\frac{\pi}{\delta_{\mathrm{SC}}}\left[\frac{2}{3} \frac{\Delta C}{C_{N}}\left(\frac{T_{\mathrm{C}}}{T}-1\right)\right]^{1 / 2}\right\}$ 来考虑热涨落对晶 体磁性和热力学性质的影响。其中 $\Delta C / C_{N}=1.43$, $\delta_{\mathrm{SC}}=1.76$ 。从图 3(a)中磁化率的结果中可以看到, 
轨道磁矩在临界温度附近逐渐释放是导致磁化率反 常峰的物理原因。临界温度反映了晶场䢃裂能与晶 格热振动的竞争。值得一提的是, 当温度趋于绝对 零度时, 空穴数为偶数的材料磁化率下降 $(\mathrm{Tb}, \mathrm{Ho}$, $\mathrm{Tm})$, 空穴数为奇数的材料磁化率上升。这是因为偶 数空穴的 $\mathrm{RB}_{12}$ 拥有一个零磁矩的单重态的多空穴 基态，而奇数空穴的材料拥有非零磁矩的多重态的 多空穴基态。 $\mathrm{HoB}_{12}$ 之所以再次成为例外, 是因为本 计算基于立方对称假定。一旦 $\mathrm{HoB}_{12}$ 晶体在低温下 发生立方对称的破缺, 该四重态基态也会破简并成 两个二重态。人们在实验上已经观察到了类似的现象。

类似磁化率，利用公式(12)计算比热随温度的 变化。如图 3(b)所示, 在临界温度附近, 磁矩贡献的 比热也显示出一个尖锐的峰, 峰的宽度和晶场䢃裂 的大小正相关 ( $R$ 为普适气体常量)。

在物理图像中, 热振动引起的晶场能级的展宽 导致轨道磁矩逐渐释放, 这与稀土材料中磁矩幅度 调制的相变特点相吻合。可以认为, 这种磁反常来 自于稀土离子本身, 而非来自材料的某种特定磁结 构。在很多稀土组成的化合物中, 类似的现象均有 出现 ${ }^{[24-26]}$ 。

除了顺磁稀土离子的贡献之外, 实验测量中还 包含了声子和传导电子 ${ }^{[11-12]}$ 的贡献。尽管很难和实 验进行定量比较, 本研究提取了涉及磁化率和比热 反常峰的跃变值进行比对。在表 3 中可以看到,
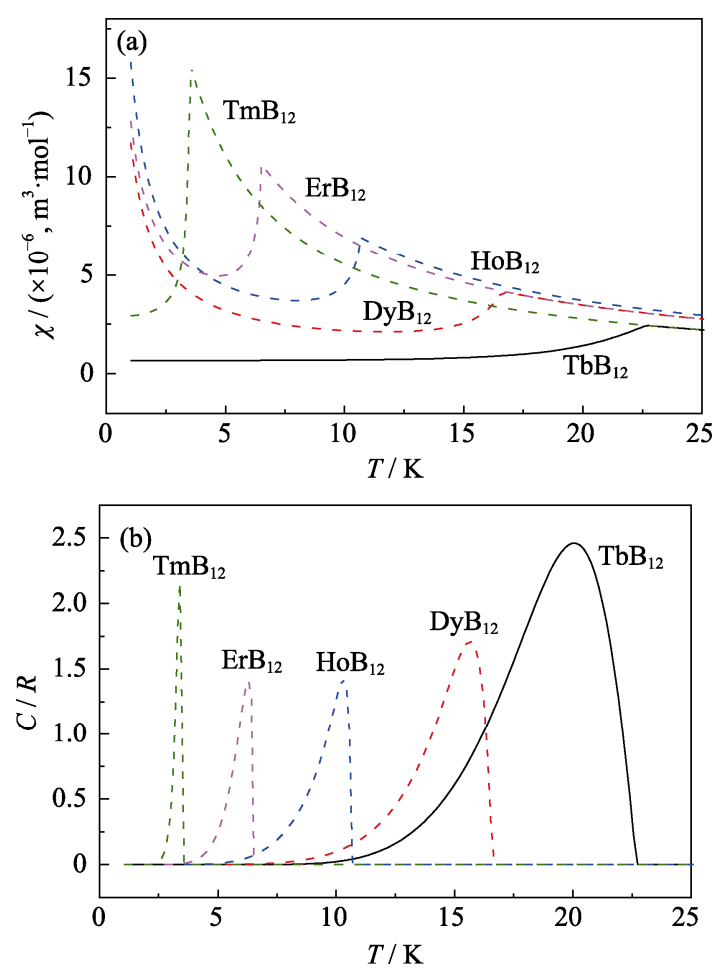

图 $3 \mathrm{RB}_{12}$ 的磁化率 $(\mathrm{a})$ 和比热(b)随温度变化曲线

Fig. 3 Magnetic susceptibilities (a) and specific heat (b) of
$\mathrm{RB}_{12}$ as functions of temperature

表 3 磁性反常附近 $\mathbf{R B}_{12}$ 的磁化率 $\left(\times 10^{-6}, \mathrm{~m}^{3} \cdot \mathrm{mol}^{-1}\right)$ 和比热跃变数据

Table 3 Magnetic susceptibility and specific heat jumps near the magnetic anomaly of $\mathbf{R B}_{12}\left(\times 10^{-6}, \mathrm{~m}^{3} \cdot \mathrm{mol}^{-1}\right)$

\begin{tabular}{ccccc}
\hline $\mathrm{RB}_{12}$ & $\Delta \chi($ Exp. $)$ & $\Delta \chi($ Cal. $)$ & $\Delta C / R($ Exp. $)$ & $\Delta C / R($ Cal. $)$ \\
\hline $\mathrm{TbB}_{12}$ & - & 2.39 & 1.4 & 2.46 \\
$\mathrm{DyB}_{12}$ & 3.89 & 4.15 & 1.2 & 1.72 \\
$\mathrm{HoB}_{12}$ & 5.65 & 6.91 & - & 1.43 \\
$\mathrm{ErB}_{12}$ & 6.91 & 10.56 & 1.1 & 1.41 \\
$\mathrm{TmB}_{12}$ & 4.77 & 15.58 & 2.0 & 2.16 \\
\hline
\end{tabular}

除了较重的稀土外, 估算值和实验值 ${ }^{[11-12]}$ 的量级 一致。

\section{2 中子散射}

在这一节中, 对引言中提到的 $\mathrm{RB}_{12}$ 低温中子衍 射谱上位于 $(1 / 2,1 / 2,1 / 2)$ 附近的双峰结构做出了解 释。很多研究人员指出, 在低温下稀土离子并不处 于 $\mathrm{B}_{24}$ 笼子的几何中心 ${ }^{[9-10]}$ 。他们的计算结果显示, 在笼子内部存在多对满足立方对称的双势阱。局域 Jahn-Taller 效应保证了这种双势阱的存在。双势阱 包含了两种爱因斯坦声子模式，低频模式 [27-28] $(5 \mathrm{meV})$ 对应每个势阱底部的局部曲率，高频模式 ${ }^{[12]}$ $(20 \mathrm{meV})$ 对应双势阱的整体曲率。低温下自发的 Jahn-Teller 效应导致稀土离子二聚化和晶体结构的 扩胞。双峰中的第一个峰可能来自衍射面指数(111) 方向的扩胞，第二个峰可能来自于低频爱因斯坦振 动模式对中子造成的非弹性散射。利用布拉格散射 条件 $2 d \sin \theta=n \lambda$, 可以得到第一个峰位于 $2 \theta=15.95^{\circ}$ 处; 而广义布拉格散射公式 $\sin \theta_{1} / \lambda+$ $\sin \theta_{2} / \lambda^{\prime}=\sqrt{h^{2}+k^{2}+l^{2}} / a$ 给出中子在非弹性散射 前后的能量分别为 14.2 和 $9.2 \mathrm{meV}$, 对应散射角(入 射和出射角之和)为 $17.51^{\circ}$ 或 $17.90^{\circ}$, 组合成了双峰 结构中的后者。而在高温时, 双势阱组态的随机占 据使得晶体在统计上又回到了立方对称的面心立方 结构。这和实验一致, 这时双峰在临界温度以上自 然就消失了。

\section{3 结论}

本工作研究了一系列稀土一嗍十二化合物 $\mathrm{RB}_{12}$ $(\mathrm{R}=\mathrm{Tb}, \mathrm{Dy}, \mathrm{Ho}, \mathrm{Er}, \mathrm{Tm})$ 在 22 3 K 范围内磁性和比热 的反常现象, 研究结果表明稀土离子微小的晶场䢃 裂能易受热涨落的影响。将晶场䢃裂能视作一个热 力学序参量, 而不仅仅是一个量子力学参量。反常现 象源自热振动引起的晶场能级展宽和由此导致的 $4 \mathrm{f}$ 
电子轨道磁矩在临界温度附近逐渐释放。本工作的 模型磁导率和比热反常峰给出了合理的解释, 说明 了偶数空穴和奇数空穴稀土离子系统的不同低温磁 学行为。基于稀土离子在 $\mathrm{B}_{24}$ 笼子中的双势阱结构, 利用晶格的二聚化和非弹性散射, 对中子衍射谱中 出现的双峰结构也给出了一种可能的解释。本研究 的结论可通过临界温度以下的 $X$ 射线衍射实验是否 出现单峰来判断。

\section{参考文献:}

[1] FLACHBART K, ALEKSEEV P, GRECHNEV G, et al. Rare Earths: Research and Applications. Nova Science Publishers, 2008: 79.

[2] MORI T. Handbook on the Physics and Chemistry of Rare Earths. Amsterdam: Elsevier, 2008: 105-173.

[3] TEYSSIER J, LORTZ R, PETROVIC A, et al. Effect of electron-phonon coupling on the superconducting transition temperature in dodecaboride superconductors: a comparison of $\mathrm{LuB}_{12}$ with $\mathrm{ZrB}_{12}$. Physical Review B, 2008, 78(13): 134504.

[4] OKAMURA H, KIMURA S, SHINOZAKI H, et al. Optical conductivity of the Kondo insulator $\mathrm{YbB}_{12}$ : gap formation and low-energy excitations. Physical Review B, 1998, 58(12): R7496.

[5] BERTAUT F, BLUM P. Structure of uranium borides. CR Acad. Sci.(Paris), 1949, 229: 666-667.

[6] LA PLACA S, BINDER I, POST B. Binary dodecaborides. Journal of Inorganic and Nuclear Chemistry, 1961, 18: 113-117.

[7] MENUSHENKOV A P, YAROSLAVTSEV A A, ZALUZHNYY I A, et al. Features of the local structure of rare-earth dodecaborides $\mathrm{RB}_{12}(\mathrm{R}=\mathrm{Ho}, \mathrm{Er}, \mathrm{Tm}, \mathrm{Yb}, \mathrm{Lu})$. JETP letters, 2013, 98(3): $165-169$.

[8] VORONOVICH D, TARAN A, SHITSEVALOVA N, et al. Anisotropy of the erbium dodecaboride thermionic properties. Solid State Sciences, 2012, 14(11/12): 1624-1628.

[9] WERHEIT H, FILIPOV V, SHIRAI K, et al. Raman scattering and isotopic phonon effects in dodecaborides. Journal of Physics: Condensed Matter, 2011, 23(6): 065403.

[10] MOISEENKO L L, ODINTSOV V V. The magnetic properties of rare earth dodecaborides. Journal of the Less Common Metals, 1979, 67(1): 237-243.

[11] GABÁNI S, BAT'KO I, FLACHBART K, et al. Magnetic and transport properties of $\mathrm{TmB}_{12}, \mathrm{ErB}_{12}, \mathrm{HoB}_{12}$ and $\mathrm{DyB}_{12}$. Journal of Magnetism and Magnetic Materials, 1999, 207(1/2/3): 131-136.

[12] CZOPNIK A, SHITSEVALOVA N, KRIVCHIKOV A, et al. Thermal properties of rare earth dodecaborides. Journal of Solid State Chemistry, 2004, 177(2): 507-514.

[13] GUBBENS P C M, VAN DER KRAAN A M, BUSCHOW K H J. $\mathrm{TmB}_{12}$ : another singlet-triplet induced moment system. Physica $B^{+}$ C, 1985, 130(1/2/3): 412-414.
[14] BAŤKO I, FLACHBART K, MAŤAŠ S, et al. Magnetic phase transitions in $\mathrm{TmB}_{12}$ and $\mathrm{HoB}_{12}$. Journal of Alloys and Compounds, 1993, 196(1/2): 133-135.

[15] SLUCHANKO N E, BOGACH A V, GLUSHKOV V V, et al. Anomalies of magnetoresistance of compounds with atomic clusters $\mathrm{RB}_{12}(\mathrm{R}=\mathrm{Ho}, \mathrm{Er}, \mathrm{Tm}, \mathrm{Lu})$. Journal of Experimental and Theoretical Physics, 2009, 108(4): 668-687.

[16] JÄGER B, PALUCH S, ŻOGAŁ O, et al. Characterization of the electronic properties of $\mathrm{YB}_{12}, \mathrm{ZrB}_{12}$, and $\mathrm{LuB}_{12}$ using ${ }^{11} \mathrm{~B} \mathrm{NMR}$ and first-principles calculations. Journal of Physics: Condensed Matter, 2006, 18(8): 2525.

[17] SIEMENSMEYER K, HABICHT K, LONKAI T, et al. Magnetic properties of the frustrated fcc-antiferromagnet $\mathrm{HoB}_{12}$ above and below $T_{\mathrm{N}}$. Journal of Low Temperature Physics, 2007, 146(5/6): 581-605.

[18] MURASIK A, CZOPNIK A, KELLER L, et al. Unconventional magnetic behaviour of $\mathrm{TbB}_{12}$ at low temperature. Physica Status Solidi (b), 2002, 234(3): R13-R15.

[19] KOHOUT A, BATKO I, CZOPNIK A, et al. Phase diagram and magnetic structure investigation of the fcc antiferromagnet $\mathrm{HoB}_{12}$. Physical Review B, 2004, 70(22): 224416.

[20] HUTCHINGS M T. Point-charge Calculations of Energy Levels of Magnetic Ions in Crystalline Electric Fields. New York: Academic Press, 1964: 227-273.

[21] FREEMAN A J, WATSON R. Theoretical investigation of some magnetic and spectroscopic properties of rare-earth ions. Physical Review, 1962, 127(6): 2058.

[22] ODINTSOV V V. Influence of the crystalline structure on the mechanical properties of dodecaborides of rare-earth metals and zirconium. Materials Science, 2016, 51(4): 576-582.

[23] FULDE P, LOEWENHAUPT M. Magnetic excitations in crystal-field split 4f systems. Advances in Physics, 1985, 34(5): 589-661.

[24] HU JING-SAN, LI GUAN-NAN, HUANG XIAO-KUN, et al. Role of the crystal electric field on the two magnetic transitions in the orthorhombic $\mathrm{YbMnO}_{3}$ perovskite. Physical Review B, 2019, 99(13): 134418.

[25] MALKIN B Z, BUD'KO S L, NOVIKOV V V. Crystal-field approach to rare-earth higher borides: dimerization, thermal, and magnetic properties of $\mathrm{RB}_{50}(\mathrm{R}=\mathrm{Tb}$, Dy, Ho, Er, Tm). Physical Review Materials, 2020, 4(5): 054409.

[26] NOVIKOV V V, ZHEMOEDOV N A, MATOVNIKOV A V, et al. Specific features of thermal and magnetic properties of $\mathrm{YbB}_{50}$ at low temperatures. Physical Review Materials, 2018, 2(5): 054401.

[27] BOUVET A, KASUYA T, BONNET M, et al. Magnetic excitations observed by means of inelastic neutron scattering in polycrystalline. Journal of Physics: Condensed Matter, 1998, 10(25): 5667.

[28] NEFEOdOVA E V, ALEKSEEV P A, MIGNOT J M, et al. Inelastic neutron scattering study of the Kondo semiconductor $\mathrm{YbB}_{12}$. Physical Review B, 1999, 60(19): 1350. 